\title{
CALCULATION OF THE POTENTIAL FIELD IN NERVE STIMULATION USING A MULTIGRID METHOD
}

\author{
Rudolf Hoekema, Johannes J. Struijk, Cornelis H. Venner, \\ Eleanor V. Goodall and Jan Holsheimer \\ Inst. of Biomed. Technol., Dept. Electr. Eng., University of Twente, P.O. box 217, \\ 7500 AE Enschede, the Netherlands
}

\begin{abstract}
This paper deals with the first step in the modeling of nerve stimulation: the calculation of the potential field in a 3D volume conductor model of the nerve, surroundings and electrodes. Because of its time efficiency, a multigrid method was used to calculate the field. Compared to a Gauss-Seidel relaxation (overrelaxation factor $=1.7$ ), a calculation time reduction of a factor 20 was obtained.
\end{abstract}

\section{INTRODUCTION}

Nerve stimulation modeling is usually carried out in two steps. First the potential distribution in a volume conductor due to stimulation has to be calculated. The obtained field is subsequently applied to a (McNeal) model of a nerve fiber. Thus, the behaviour of a nerve fiber in a specific volume conductor model can be calculated [1].

The present contribution deals with the calculation of the potential field in a $3 \mathrm{D}$ volume conductor model. In such a model several compartments are present, each representing a different part of the environment in which the nerve fiber is situated (such as the fascicle, the perineurium, the epineurium, the surrounding fluid). Each compartment has a specific (anisotropic) conductivity. The potential field can be calculated by solving Poisson's equation with inhomogeneous coefficients:

$$
\nabla . \sigma \nabla u=f
$$

where $u$ is the potential, $\sigma$ is the conductivity tensor and $f$ represents the current sources in the model. A more general notation of Poisson's equation is $L u=f$. For a 3D configuration with varying conductivity, (1) can not be solved analytically. Hence a numerical approach is needed, in which the equation is first discretized using Taylor expansions. For example, the ID discretized version of (1) is:

$$
\frac{\sigma_{i} u_{i-1}-\left(\sigma_{i}+\sigma_{i+1}\right) u_{i}+\sigma_{i+1} u_{i+1}}{h^{2}}=f_{i}
$$

where $u_{i}$ is the potential at point $i, \sigma_{i}$ is the conductivity between point $i$ and $i-1$ and $f_{i}$ is the source term at point $i$. Secondly, the resulting set of equations is solved, using for example an iterative scheme like the pointwise Gauss-Seidel relaxation scheme, which involves subsequently replacing the value of $u_{i}$ for all points with $u_{\mathrm{i}}$ according to (2). The computing time will strongly depend on the number of nodes. For example, Gauss-Seidel relaxation requires $O\left(N^{2}\right.$ $\log (\mathrm{N})$ ) operations to obtain a converged solution, if $\mathrm{N}$ is the number of points. In the problem at hand, we use $N=57^{3}=185193$ points. Using Gauss-Seidel relaxation, this will lead to excessive computing times.

\section{MULTIGRID}

The concept of a multigrid solver for a partial differential problem lies in the convergence behavior of most iterative schemes, in which each point is updated using the solution on neighboring points only. As a result, high frequency (local) error components are reduced very fast. However, smooth components are hardly affected by this scheme and require many iterations to converge, yielding computing times as indicated above. Hence, after the first few iterations non-smooth errors are effectively reduced, but a smooth error remains and convergence is very slow.

In a multigrid solver this slowness is overcome using the smoothness of the error after the first sweeps. Instead of continuing the iteration on the fine grid, a coarse grid is used to calculate the solution of the error. This can be done quite efficiently, because much fewer points exist on this grid and the smooth error will be less smooth on the coarse grid and therefore more efficiently reduced. Once an accurate approximation to the error is obtained, it is used to correct the solution on the fine grid. This entire concept is applied recursively and consequently, an entire set of grids is used to accelerate convergence. This so-called Correction Cycle is the basic element in a muitigrid solver and has an overall convergence speed that is mesh-size independent.

In addition the coarser grids can be used to generate an accurate first approximation on the finest grid. The resulting algorithm, generally referred to as Full Multigrid, yields a converged solution in $O(N)$ operations.

Schematically, the multigrid algorithm can be described as follows [2]:

1. Perform a few relaxation sweeps on the fine grid.

2. Derive an expression for the smooth error problem and transfer it to the coarse grid.

3. Calculate the coarse grid correction. 
4. Transfer the coarse grid correction to the fine grid and correct the fine grid solution with it.

5. Perform a few relaxation sweeps on the corrected solution on the fine grid.

Each element in this scheme is briefly explained below. For simplicity only 2 grids are assumed, with grid size $h$ and H. Generally, $\mathrm{H}=2 \mathrm{~h}$.

Step 1: Pre-relaxation

First, a few relaxation sweeps are carried out on the fine grid to obtain an initial approximation of the solution. The relaxation on the fine grid of $L^{\mathrm{b}} u^{\mathrm{k}}=f^{\mathrm{h}}$ is done using the Gauss-Seidel method as described in the introduction.

Step 2: Derivation of smooth problem expression and transfer to coarse grid

The (smooth) error in the solution on the fine grid is defined as $e^{b}=u^{h}-u^{h}$ where $u^{h}$ is the exact discretized solution. If Poisson's operator $L^{\mathrm{h}}$ is applied to both sides, $L^{\mathrm{h}} e^{\mathrm{h}}=$ $L^{h}\left(u^{h}-u^{h}\right)=f^{h}-L^{h} u^{h}$, is obtained. The right hand side is the residual $r^{b}$. If this expression is solved completely, $e^{t}$ can be added to $u^{h}$, resulting in $u^{h}$. The equation $L^{h} e^{k}=r^{h}$ is therefore a suitsble smooth probiem expression.

The residual is transferred to the coarse grid by the restriction operator $I \stackrel{H}{h}$. This operator averages the residuals on the points surrounding the coarse grid point using the conductivities between these points [3]. In short, $r^{H}=I_{b}^{\mathrm{H}} r^{b}$.

Step 3. Calculation of coarse grid correction

Now, the coarse grid problem $L^{\mathrm{H}} e^{\mathrm{H}}=r^{\mathrm{H}}$ has to be solved, whether recursively or by pointwise relaxation. The coefficients of $L^{\mathrm{H}}$ are derived by weighted averaging of the fine grid coefficients between two coarse grid points [3].

Step 4. Transfer of coarse grid correction to fine grid and correction of fine grid solution with it.

The coarse grid correction is now transferred to the fine grid with interpolation operator $I_{\mathrm{H}}^{\mathrm{h}}$. The error $e^{\mathrm{h}}$ on fine grid points coinciding with coarse grid points is taken directly from the coarse grid points; on the other fine grid points, $e^{b}$ is interpolated from the neighboring coarse grid points [3]. The error is simply added to $u^{h}$. In short: $u^{b}=u^{b}+l{ }_{\mathrm{H}}^{\mathrm{b}} e^{\mathrm{b}}$.

\section{Step 5: Post-relaxation}

Finally, a few relaxation sweeps are carried out on the to reduce non-smooth errors in the solution originating from the interpolation of the coarse grid correction.

\section{RESULTS}

The multigrid method briefly described above was implemented in a solver for the potential field in a volume conductor describing a model of a fascicle of the N. Peroneus of the rabbit with a cuff electrode [4] around it, as shown in Figure 1. The distance between fine grid points was 50,50 and $200 \mu \mathrm{m}$ in the $x, y$ and $z$-directions. Conductivity-changes of about a factor 1000 were present. A tripolar electrode with a transverse anode was used. The currents at the contacts were $0.2,-1.0$ and $0.2 \mathrm{~mA}$ (tripolar part) and $0.6 \mathrm{~mA}$ (transverse anode). Electrode contacts were modeled as point currents sources.

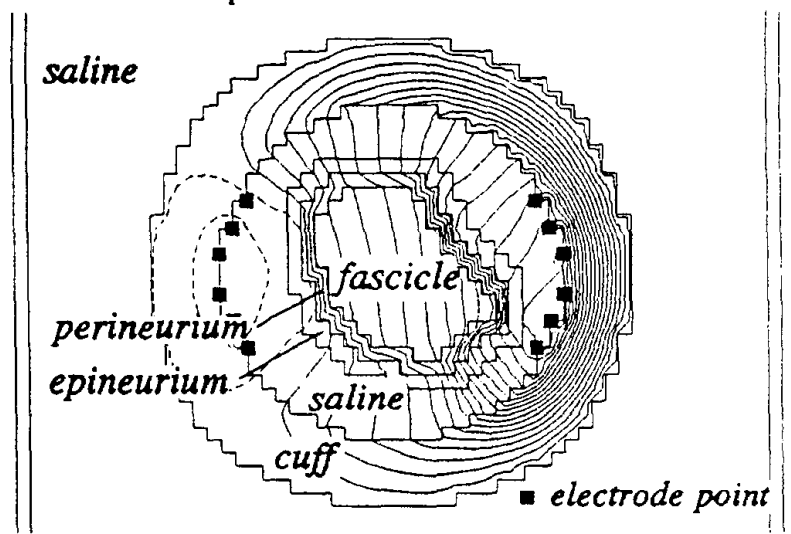

Figure 1. Transverse secrion through center of $3 D$ volume conductor madel describing a fascicle and cuff electrode. The potenrial field was calculaved using a mulrigrid algorithm. Currents at conlacts were: anode (left) $0.6 \mathrm{~mA}$, calhode (right) $-1.0 \mathrm{~mA}$. The iso-potential lines are $60 \mathrm{mV}$ apart. Two other anodes are in higher and lower sections.

The time needed to calculate this potential field on a HP9000-730 workstation was 60 seconds. Using the GaussSeidel relaxation method with an overrelaxation factor of 1.7 , a field with the same accuracy was obtained after 20 minutes. Thus a calculation time reduction of a factor 20 was obtained. This reduction factor will increase with $O(N$ $\log (\mathrm{N})$ ) when a grid with more points is used.

\section{REFERENCES}

[1] Struijk, J.J., "Immediate Effects of Spinal Cord Stimulation", 1992, Thesis, University of Twente, the $\mathrm{Ne}$ therlands.

[2] Brandt, A, "Multigrid Techniques: 1984 Guide with Applications to Fluid Dynamics", 1984, Gesellsch. Für Mathematik und Datenverarbeitung, Bonn

[3] Alcouffe, R.E., et al., "The Multigrid method for the diffusion equation with strongly discontinuous coefficients", 1981, SIAM. J. Sci. Stat. Comput. vol. 2, pp.430-454.

[4] Sweeney, J.D., et al., "A Nerve Cuff Technique for Selective Excitation of Peripheral Nerve Trunk Regions", 1990, IEEE Trans. BME, vol. 37, pp 706-715. 